\title{
FIRST DETECTION OF LINEAR POLARIZATION IN THE LINE PROFILES OF ACTIVE COOL STARS*
}

\author{
O. Kochukhov ${ }^{1}$, V. Makaganiuk ${ }^{1}$, N. Piskunov ${ }^{1}$, F. Snik ${ }^{2}$, S. V. Jefferrs ${ }^{2}$, C. M. Johns-Krull ${ }^{3}$, C. U. Keller ${ }^{2}$, \\ M. RoDENHUIS ${ }^{2}$, AND J. A. VALENTI ${ }^{4}$ \\ ${ }^{1}$ Department of Physics and Astronomy, Uppsala University, Box 516, Uppsala 75120, Sweden; oleg.kochukhov@ fysast.uu.se \\ 2 Sterrekundig Instituut, Universiteit Utrecht, Box 80000, 3508 TA Utrecht, The Netherlands \\ ${ }^{3}$ Department of Physics and Astronomy, Rice University, 6100 Main Street, Houston, TX 77005, USA \\ ${ }^{4}$ Space Telescope Science Institute, 3700 San Martin Dr, Baltimore, MD 21211, USA \\ Received 2011 March 1; accepted 2011 March 30; published 2011 April 13
}

\begin{abstract}
The application of high-resolution spectropolarimetry has led to major progress in understanding the magnetism and activity of late-type stars. During the last decade, magnetic fields have been discovered and mapped for many types of active cool stars using spectropolarimetric data. However, these observations and modeling attempts are fundamentally incomplete since they are based on the interpretation of the circular polarization alone. Taking advantage of the newly built HARPS polarimeter, we have obtained the first systematic observations of several cool active stars in all four Stokes parameters. Here we report the detection of magnetically induced linear polarization for the primary component of the very active RS CVn binary HR 1099 and for the moderately active K dwarf $\varepsilon$ Eri. For both stars the amplitude of linear polarization signatures is measured to be $\sim 10^{-4}$ of the unpolarized continuum, which is approximately a factor of 10 lower than for circular polarization. This is the first detection of the linear polarization in line profiles of cool active stars. Our observations of the inactive solar-like star $\alpha$ Cen A show neither circular nor linear polarization above the level of $\sim 10^{-5}$, indicating the absence of a net longitudinal magnetic field stronger than $0.2 \mathrm{G}$.
\end{abstract}

Key words: polarization - stars: activity - stars: individual (HR 1099, $\varepsilon$ Eri, $\alpha$ Cen A) - stars: late-type - stars: magnetic field

Online-only material: color figure

\section{INTRODUCTION}

Polarization in spectral lines is the most direct and reliable signature of the presence of magnetic fields on the surfaces of stars. While the Zeeman broadening of unpolarized spectra can be used, with some ambiguity, to diagnose kG-strength fields in extremely active stars (Guenther et al. 1999; Johns-Krull 2007), circular polarimetry enables detection of global magnetic fields on the order of $1 \mathrm{G}$ and weaker in moderately active stars (Aurière et al. 2009, 2010; Lignières et al. 2009).

The diagnostic power of polarimetry comes from the possibility of measuring very precisely magnetic signals in spectral lines against an unpolarized continuum and boosting the signalto-noise ratio $(\mathrm{S} / \mathrm{N})$ by combining weak polarization signals in thousands of spectral lines recorded simultaneously with a wide wavelength coverage échelle spectrometer (Donati et al. 1997). Furthermore, Doppler imaging analysis of the rotational modulation of circular polarization in spectral lines has resulted in detailed maps of stellar magnetic fields (e.g., Donati et al. 2003), leading to many new insights into the magnetism and activity of different groups of late-type stars (Donati \& Landstreet 2009).

Despite spectacular results of the recent investigations, spectropolarimetry of stellar magnetic fields remains fundamentally limited. Most importantly, high-resolution spectropolarimetric observations of all late-type stars and of the majority of earlytype stars are limited to circular polarization (Stokes $V$ ). A significantly weaker linear polarization (Stokes $Q$ and $U$ ) signal due to the transverse Zeeman effect has been detected and used for diagnosing stellar magnetic topologies only for a few strongly magnetic Ap stars (Wade et al. 2000; Kochukhov et al. 2004)

\footnotetext{
* Based on observations obtained at the European Southern Observatory (ESO programs 083.D-1000(A) and 084.D-0338(A)).
}

and for one magnetic white dwarf (Valyavin et al. 2008). Previous spectropolarimetric observations of late-type stars in the Stokes $Q$ and $U$ parameters were limited to the objects where the presence of a relatively strong linear polarization was unrelated to magnetic fields (e.g., Boyle et al. 1986; Vink et al. 2003).

Incomplete Stokes parameter data sets typically employed for magnetic stars are plagued by a reduced sensitivity to the horizontal magnetic field component and, in general, do not allow a unique reconstruction of the stellar magnetic field geometries (Kochukhov \& Piskunov 2002). Four Stokes parameter studies of Ap stars also demonstrated that magnetic models based on the interpretation of circular polarization alone miss the smaller-scale magnetic structures (Kochukhov \& Wade 2010).

High-resolution, full four Stokes parameter observations of active cool stars represent a crucial step toward more robust and complete analysis of their magnetic field topologies. Here we present the first results of simultaneous circular and linear polarization observations of active stars with a new polarimetric device: HARPSpol.

\section{SPECTROPOLARIMETRIC OBSERVATIONS}

The HARPS spectrometer (Mayor et al. 2003), fed by the $3.6 \mathrm{~m}$ telescope at the European Southern Observatory (ESO), is one of the most powerful astronomical tools for precision studies of stellar spectra. It is the highest resolution ESO spectrograph, providing a coverage of the $380-690 \mathrm{~nm}$ wavelength region in a single exposure with the resolving power of $\lambda / \Delta \lambda=115,000$ and a long-term velocity stability of better than $1 \mathrm{~m} \mathrm{~s}^{-1}$.

The new polarimeter, HARPSpol (Snik et al. 2011; Piskunov et al. 2011), is installed in the Cassegrain focus of the $3.6 \mathrm{~m}$ 
Table 1

Journal of the HARPSpol Four Stokes Parameter Observations of Late-type Stars

\begin{tabular}{llcccccccc}
\hline \hline Name & $\begin{array}{c}\text { Date } \\
\text { (UT) }\end{array}$ & $\begin{array}{c}\text { HJD } \\
(2,400,000+)\end{array}$ & $\begin{array}{c}\text { Stokes } \\
\text { Parameter }\end{array}$ & $\begin{array}{c}t_{\exp } \\
(\mathrm{s})\end{array}$ & $\mathrm{S} / \mathrm{N}$ & $\begin{array}{c}\sigma_{\text {LSD }} \\
\left(10^{-5} I_{\mathrm{c}}\right)\end{array}$ & $\begin{array}{c}P_{\max } \\
\left(10^{-5} I_{\mathrm{c}}\right)\end{array}$ & $\begin{array}{c}\text { FAP } \\
\text { Det. }\end{array}$ & $\begin{array}{c}\text { Deg } \\
\text { Flag }\end{array}$ \\
\hline$\alpha$ Cen A & 2009 Jun 2 & 54984.6493 & $V$ & $72 \times 10$ & 3410 & 0.87 & $<1.92$ & $9.9 \mathrm{E}-01$ & ND \\
& 2009 Jun 2 & 54984.6915 & $Q$ & $72 \times 10$ & 2630 & 0.69 & $<1.34$ & $6.9 \mathrm{E}-01$ & ND \\
& 2009 Jun 2 & 54984.7380 & $U$ & $96 \times 10$ & 3010 & 0.60 & $<1.19$ & $9.8 \mathrm{E}-01$ & ND \\
\hline HR 1099 & 2010 Jan 4 & 55200.6197 & $V$ & $4 \times 421$ & 390 & 4.88 & 190.9 & $<1.0 \mathrm{E}-16$ & DD \\
& 2010 Jan 4 & 55200.6441 & $Q$ & $4 \times 421$ & 360 & 2.88 & 15.1 & $<1.0 \mathrm{E}-16$ & DD \\
& 2010 Jan 4 & 55200.6715 & $U$ & $4 \times 421$ & 370 & 2.81 & 11.0 & $7.2 \mathrm{E}-08$ & DD \\
& 2010 Jan 16 & 55212.5897 & $V$ & $4 \times 421$ & 440 & 4.24 & 92.0 & $<1.0 \mathrm{E}-16$ & DD \\
& 2010 Jan 16 & 55212.5458 & $Q$ & $4 \times 421$ & 420 & 2.44 & 12.1 & $<1.0 \mathrm{E}-16$ & DD \\
& 2010 Jan 16 & 55212.5675 & $U$ & $4 \times 421$ & 420 & 2.44 & 11.6 & $1.8 \mathrm{E}-14$ & DD \\
\hline$\varepsilon$ Eri & 2010 Jan 8 & 55204.5758 & $V$ & $4 \times 250$ & 880 & 1.99 & 16.4 & $<1.0 \mathrm{E}-16$ & DD \\
& 2010 Jan 8 & 55204.5434 & $Q$ & $4 \times 250$ & 880 & 1.20 & 4.47 & $2.7 \mathrm{E}-05$ & MD \\
& 2010 Jan 8 & 55204.5605 & $U$ & $4 \times 250$ & 880 & 1.15 & 2.42 & $8.2 \mathrm{E}-01$ & ND \\
& 2010 Jan 9 & 55205.6000 & $V$ & $4 \times 240$ & 820 & 2.22 & 46.9 & $<1.0 \mathrm{E}-16$ & DD \\
& 2010 Jan 9 & 55205.5714 & $Q$ & $4 \times 240$ & 860 & 1.22 & 3.85 & $1.9 \mathrm{E}-05$ & MD \\
& 2010 Jan 9 & 55205.5853 & $U$ & $4 \times 240$ & 820 & 1.25 & 5.49 & $2.0 \mathrm{E}-05$ & MD \\
& 2010 Jan 11 & 55207.6232 & $V$ & $4 \times 240$ & 770 & 2.39 & 33.0 & $<1.0 \mathrm{E}-16$ & DD \\
& 2010 Jan 11 & 55207.5949 & $Q$ & $4 \times 240$ & 800 & 1.32 & 5.81 & $1.4 \mathrm{E}-10$ & DD \\
& 2010 Jan 11 & 55207.6086 & $U$ & $4 \times 240$ & 780 & 1.33 & 2.81 & $7.9 \mathrm{E}-01$ & ND \\
\hline
\end{tabular}

Note. ND, MD, and DD in the last column indicate no, marginal, and definite detections of polarization signature.

telescope, feeding the two existing HARPS optical fibers. The polarimeter consists of two separate polarimetric units: one for circular and another for linear polarization measurements. Each unit is equipped with a modulator - a superachromatic quarterwave or half-wave plate. The light is split into two orthogonally polarized beams by a polarizing beam splitter (Foster prism). The corresponding spectra are recorded simultaneously on the $4 \mathrm{k} \times 4 \mathrm{k}$ CCD mosaic. A slider inserts the polarimeter from a completely retracted position, and allows for selection of either the circular or the linear polarimeter.

The two orthogonally polarized beams injected into the fibers can be exchanged by rotating the half-wave plate with the increment steps of $45^{\circ}$ and the quarter-wave plate with $90^{\circ}$ steps. A combination of exposures obtained with different modulator orientations allows compensation of, to first order, flat-fielding errors and effects of seeing and other artifacts (Semel et al. 1993). The high spectral resolution of HARPSpol is especially useful for detecting complex and weak linear polarization signatures in cool stars (Semel et al. 2009).

Each of our polarization observations of cool stars consisted of four sub-exposures, which were calibrated and processed with the help of the REDUCE package (Piskunov \& Valenti 2002). The resulting one-dimensional spectra were demodulated using the "ratio" method described by Bagnulo et al. (2009) and re-normalized to the continuum intensity, yielding a spectrum in one of the Stokes parameters $\left(V / I_{\mathrm{c}}, Q / I_{\mathrm{c}}\right.$, or $\left.U / I_{\mathrm{c}}\right)$, the corresponding intensity spectrum $\left(I / I_{\mathrm{c}}\right)$, associated error bars, and a diagnostic null spectrum. The circular to linear polarization cross-talk is estimated to be below $0.5 \%$ using symmetry properties of the Stokes profiles for the slowly rotating Ap star $\gamma$ Equ.

Table 1 summarizes the four Stokes parameter observations of three late-type stars-HR 1099, $\varepsilon$ Eri, $\alpha$ Cen A-obtained during our HARPSpol runs in 2009 May and 2010 January. The first six columns in the table give the target name, the observation date, the heliocentric Julian date, the $V, Q$, and $U$ Stokes parameters, the exposure time, and the peak $\mathrm{S} / \mathrm{N}$ per $0.8 \mathrm{~km} \mathrm{~s}^{-1}$ velocity bin.

\section{MULTILINE POLARIZATION ANALYSIS}

Polarization signatures are typically not detectable in individual spectral lines of active late-type stars. Among the targets observed in our program, only HR 1099 shows evidence of the circular polarization signal in a few strong, magnetically sensitive spectral lines. To detect and characterize magnetic fields of the target stars we use a multiline technique known as leastsquares deconvolution (LSD; Donati et al. 1997). This method approximates the stellar spectrum with a superposition of identical profiles, centered at the position of individual lines and scaled according to the line's strength and magnetic sensitivity. A mean profile extracted by LSD combines common information from thousands of spectral lines, boosting the sensitivity of polarization studies.

We computed mean profiles for all Stokes parameters and the corresponding null polarization spectra using the methodology and the code developed by Kochukhov et al. (2010). The LSD line mask was based on the atomic line parameters extracted from the Vienna Atomic Line Database (VALD; Kupka et al. 1999). Depending on the star, we used between 7500 and 9000 metal lines stronger than $10 \%$ of the unpolarized continuum, achieving a multiplex gain of 30-100.

Table 1 summarizes results of the LSD analysis of our targets. Columns 7-10 give information on the noise level (per $0.8 \mathrm{~km} \mathrm{~s}^{-1}$ velocity bin) in the LSD profiles, the maximum amplitude of the LSD polarization signal, the false alarm probability (FAP) of the signal detection computed according to Donati et al. (1992), and the polarization detection flag. Following the widely used spectropolarimetric convention, we consider profiles with FAP $<10^{-5}$ as a definite detection (DD), $10^{-5}<$ FAP $<10^{-3}$ as a marginal detection (MD), and FAP $>10^{-3}$ as no detection (ND).

\section{RESULTS}

\subsection{HR 1099}

The K1 subgiant primary of the RS CVn binary HR 1099 (V711 Tau) is one of the most magnetically active late-type 

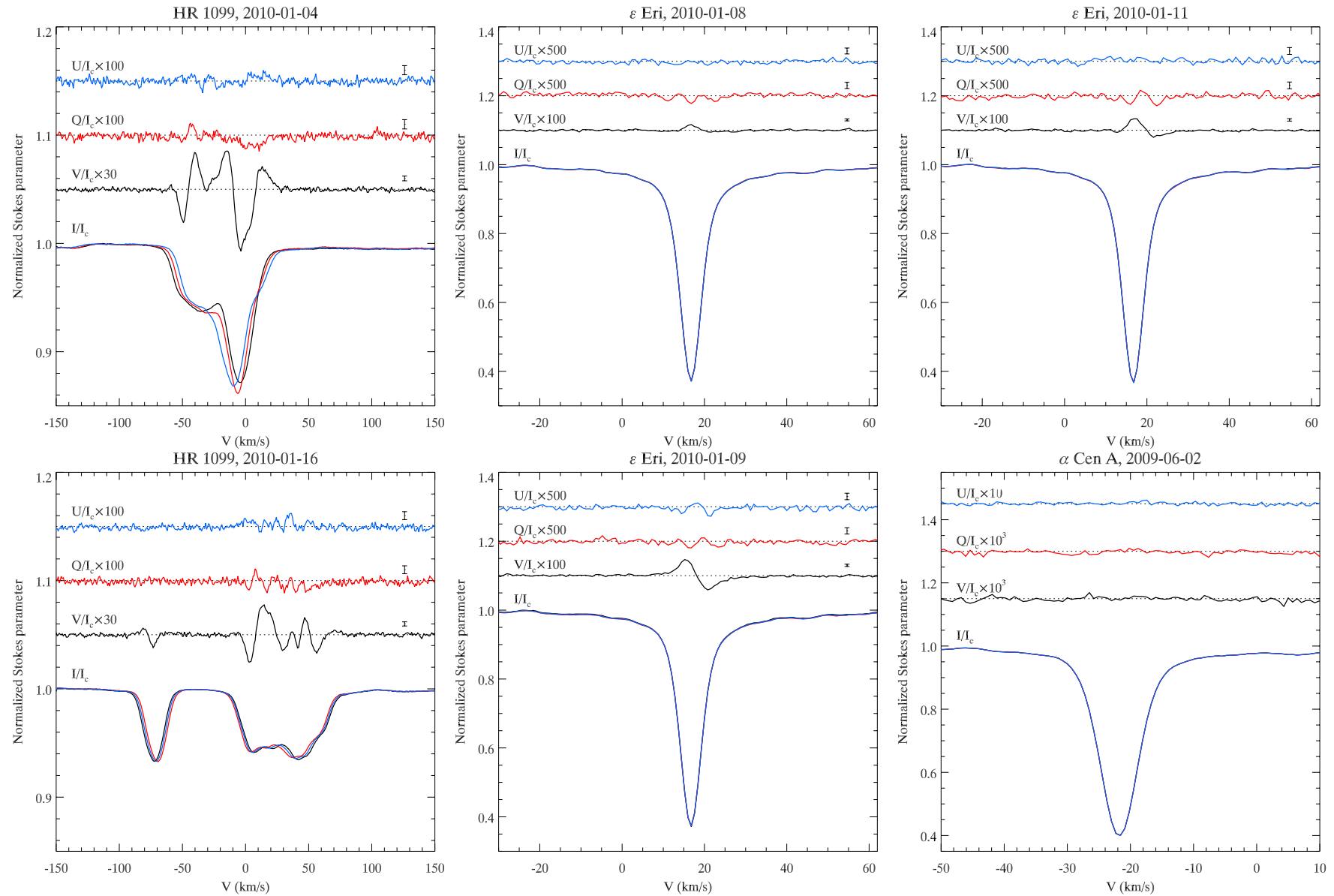

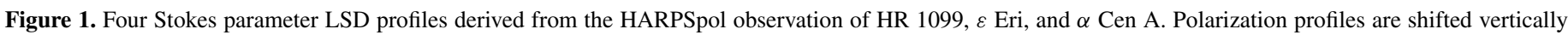
and expanded relative to the mean intensity profile. The bars on the right correspond to three times the noise level of the LSD polarization profiles.

(A color version of this figure is available in the online journal.)

stars. It was frequently studied with the Doppler imaging (Vogt et al. 1999) and circular spectropolarimetry (Donati et al. 2003). These investigations found cool spots evolving on a timescale of about one year and longer-lived magnetic field structures, dominated by regions with predominantly azimuthal field orientation.

We observed HR 1099 in all four Stokes parameters on two different nights in 2010 January. Figure 1 shows the LSD Stokes $I Q U V$ profiles obtained for this star. The contribution of both stars is evident in Stokes $I$. The distortions in the mean intensity profile of the primary indicate significant spot coverage at the time of our observations. The rapid changes of Stokes $I$ are due to the orbital motion and rotation of the primary with $P \approx 2.838$ days (Fekel 1983). The Stokes $V$ profile of the primary shows a complex field morphology. Using the first moment of LSD Stokes $V$ (Kochukhov et al. 2010), we derive a mean longitudinal magnetic field of $\left\langle B_{\mathrm{z}}\right\rangle=25.2 \pm 3.6 \mathrm{G}$ and $12.3 \pm 3.0 \mathrm{G}$ for the observations on January 4 and 16 , respectively. A weak magnetic signature in the Stokes $V$ profile of the secondary component is also clearly detected at $V \approx-70 \mathrm{~km} \mathrm{~s}^{-1}$ in the latter observation.

The $Q$ and $U$ signatures of the primary are detected in both HARPSpol observations with a very high confidence (FAP $<$ $10^{-7}$ ). This is the first detection of the linear polarization in line profiles of a cool active star. The LSD Stokes $Q U$ profiles of HR 1099 are significantly more complex than the corresponding Stokes $V$ spectra and have an amplitude of $\sim 10^{-4} I_{\mathrm{c}}$. No signal is present in the $Q$ and $U$ null profiles (FAP $>0.8$ ), confirming that detected linear polarization is intrinsic to the star and does not arise due to an instrumental or data reduction artifact.

$$
\text { 4.2. } \varepsilon \text { Eri }
$$

The K2 dwarf $\varepsilon$ Eri is one of the nearest solar-type stars and the closest exoplanet host (Hatzes et al. 2000; Benedict et al. 2006). This star is surrounded by a debris disk and shows a high level of magnetic activity. Previous studies of the Zeeman broadening in optical and infrared magnetically sensitive lines suggested the presence of fields with spatially averaged strength of 100-200 G (Valenti et al. 1995; Rüedi et al. 1997). No detection of the magnetically induced line polarization was previously reported for this star.

We observed $\varepsilon$ Eri in all four Stokes parameters during 11 nights, fully covering the 11.3 days rotation period of the star (Fröhlich 2007). Figure 1 illustrates the LSD intensity and polarization spectra of $\varepsilon$ Eri for the three selected nights. There is no evidence of variability in Stokes $I$, but a variable magnetic field is detected in the circular polarization for all nights. The Stokes $V$ LSD profiles change systematically from night to night throughout the rotation period of the star. The mean longitudinal magnetic field of $\varepsilon$ Eri varies between $-5.8 \pm 0.1$ and $4.7 \pm 0.1 \mathrm{G}$.

Despite a high $\mathrm{S} / \mathrm{N}$ of our polarimetric data, the $\operatorname{LSD} Q$ and $U$ profiles of $\varepsilon$ Eri generally do not reveal magnetic signatures. 
However, on the night of 2010 January 11 the $Q$ signature is definitely detected, while on the other two nights a marginal signal may be present in $Q$ and/or $U$ profiles. The amplitude of these linear polarization signatures does not exceed $6 \times 10^{-5} I_{\mathrm{c}}$. Rotational modulation of the linear polarization signal and its occurrence within the stellar photospheric lines suggests that polarization is unrelated to the dusty disk around $\varepsilon$ Eri.

\section{3. $\alpha \operatorname{Cen} A$}

The solar analogue $\alpha$ Cen A was observed with HARPSpol in 2009 June, during partly cloudy conditions. Nevertheless, sufficient photons were collected to reach a polarimetric sensitivity of $\sim 10^{-5}$ with the help of the LSD technique. As demonstrated by Figure 1, no signals were detected in either $V, Q$, or $U$ profiles. The corresponding null spectra are also featureless, confirming the lack of polarization artifacts down to the level of $\sim 10^{-5}$.

The non-detection of the Stokes $V$ signature corresponds to the $3 \sigma$ upper limit of $0.2 \mathrm{G}$ for the disk-averaged longitudinal magnetic field. In comparison, the net longitudinal field of the Sun reaches $1 \mathrm{G}$ during activity maximum and is below $0.1 \mathrm{G}$ during the minimum (Demidov et al. 2002). We therefore conclude that HARPSpol can detect the global magnetic fields of active solar twins. In its present low activity state, $\alpha$ Cen A appears to be reminiscent of the Sun at cycle minimum, in agreement with recent X-ray observations (Ayres 2009).

\section{DISCUSSION}

For both HR 1099 and $\varepsilon$ Eri we detected linear polarization in spectral lines with an amplitude of $(0.6-15) \times 10^{-4}$ of the unpolarized continuum, which is about a factor of 10 lower than the amplitude of the circular polarization signal recorded on the same nights. For both objects the LSD linear polarization profiles appear to be morphologically complex, requiring resolving power $\lambda / \Delta \lambda \sim 10^{5}$ for secure detection.

It is interesting to discuss the cause of linear polarization in cool stars. Solar-like spots are connected with a patchy, magnetic environment called "plage" through low-lying magnetic loops (Solanki et al. 2006). These plage regions are usually brighter than the quiet photosphere and much brighter than the cool spots, and therefore dominate the disk-integrated magnetic signal. For an on-disk active region, linear polarization can be created if the plage is not symmetrically distributed around the spot. Near the limb, the vertical components of plage can create significant linear polarization as long as there are not too many active regions such that their magnetic signals average out over the disk. The two polar regions that exhibit a weak but quite uniform outward magnetic field over a large area (Tsuneta et al. 2008) could contribute to linear polarization as well.

On the other hand, for stars with enhanced activity and nonsolar dynamo properties, such as RS CVn binaries, linear polarization can arise from the large-scale toroidal fields mapped by circular polarization Zeeman-Doppler imaging studies (Donati et al. 2003). Four Stokes parameter observations with complete rotational phase coverage are essential to confirm the reality of these azimuthal fields and distinguish different scenarios of their formation.

O.K. is a Royal Swedish Academy of Sciences Research Fellow supported by grants from the Knut and Alice Wallenberg Foundation and the Swedish Research Council.

\section{REFERENCES}

Aurière, M., Donati, J., Konstantinova-Antova, R., Perrin, G., Petit, P., \& Roudier, T. 2010, A\&A, 516, L2

Aurière, M., et al. 2009, A\&A, 504, 231

Ayres, T. R. 2009, ApJ, 696, 1931

Bagnulo, S., Landolfi, M., Landstreet, J. D., Landi Degl'Innocenti, E., Fossati, L., \& Sterzik, M. 2009, PASP, 121, 993

Benedict, G. F., et al. 2006, AJ, 132, 2206

Boyle, R. P., Aspin, C., McLean, I. S., \& Coyne, G. V. 1986, A\&A, 164, 310

Demidov, M. L., Zhigalov, V. V., Peshcherov, V. S., \& Grigoryev, V. M. 2002, Sol. Phys., 209, 217

Donati, J., \& Landstreet, J. D. 2009, ARA\&A, 47, 333

Donati, J.-F., Semel, M., Carter, B. D., Rees, D. E., \& Collier Cameron, A. 1997, MNRAS, 291, 658

Donati, J.-F., Semel, M., \& Rees, D. E. 1992, A\&A, 265, 669

Donati, J.-F., et al. 2003, MNRAS, 345, 1145

Fekel, F. C., Jr. 1983, ApJ, 268, 274

Fröhlich, H. 2007, Astron. Nachr., 328, 1037

Guenther, E. W., Lehmann, H., Emerson, J. P., \& Staude, J. 1999, A\&A, 341, 768

Hatzes, A. P., et al. 2000, ApJ, 544, L145

Johns-Krull, C. M. 2007, ApJ, 664, 975

Kochukhov, O., Bagnulo, S., Wade, G. A., Sangalli, L., Piskunov, N., Landstreet, J. D., Petit, P., \& Sigut, T. A. A. 2004, A\&A, 414, 613

Kochukhov, O., Makaganiuk, V., \& Piskunov, N. 2010, A\&A, 524, A5

Kochukhov, O., \& Piskunov, N. 2002, A\&A, 388, 868

Kochukhov, O., \& Wade, G. A. 2010, A\&A, 513, A13

Kupka, F., Piskunov, N., Ryabchikova, T. A., Stempels, H. C., \& Weiss, W. W. 1999, A\&AS, 138, 119

Lignières, F., Petit, P., Böhm, T., \& Aurière, M. 2009, A\&A, 500, L41

Mayor, M., et al. 2003, Messenger, 114, 20

Piskunov, N. E., \& Valenti, J. A. 2002, A\&A, 385, 1095

Piskunov, N., et al. 2011, Messenger, 143, 7

Rüedi, I., Solanki, S. K., Mathys, G., \& Saar, S. H. 1997, A\&A, 318, 429

Semel, M., Donati, J.-F., \& Rees, D. E. 1993, A\&A, 278, 231

Semel, M., Ramírez Vélez, J. C., Martínez González, M. J., Asensio Ramos, A., Stift, M. J., López Ariste, A., \& Leone, F. 2009, A\&A, 504, 1003

Snik, F., et al. 2011, in ASP Conf. Ser. Solar Polarization Workshop 6, ed. J. R. Kunh et al. (San Francisco, CA: ASP), in press (arXiv:1010.0397)

Solanki, S. K., Inhester, B., \& Schüssler, M. 2006, Rep. Prog. Phys., 69, 563

Tsuneta, S., et al. 2008, ApJ, 688, 1374

Valenti, J. A., Marcy, G. W., \& Basri, G. 1995, ApJ, 439, 939

Valyavin, G., Wade, G. A., Bagnulo, S., Szeifert, T., Landstreet, J. D., Han, I., \& Burenkov, A. 2008, ApJ, 683, 466

Vink, J. S., Drew, J. E., Harries, T. J., Oudmaijer, R. D., \& Unruh, Y. C. 2003, A\&A, 406, 703

Vogt, S. S., Hatzes, A. P., Misch, A. A., \& Kürster, M. 1999, ApJS, 121, 547

Wade, G. A., Donati, J.-F., Landstreet, J. D., \& Shorlin, S. L. S. 2000, MNRAS, 313,823 